\title{
无机阴离子及溶解性有机质对程海化学需氧量测定值的影响”
}

\author{
毕婷婷,杨本芹**, 罗闹, 杜娅楠,杨金凤,任 东, 顾丽鹏, 潘学军 \\ (昆明理工大学环境科学与工程学院, 昆明 650500)
}

\begin{abstract}
摘 要: 自 2005 年以来, 程海水体的化学需氧量 (COD) 持续升高, 而生化需氧量 (BOD) 却维持不变, 高镇酸盐指数 $\left(\mathrm{COD}_{\mathrm{Mn}}\right)$ 升高也较缓慢. 为研究程海 $\mathrm{COD}$ 持续升高的原因, 选取程海水体中具有代表性的无机阴离子 $\left(\mathrm{Cl}^{-} 、 \mathrm{~F}^{-} 、 \mathrm{~S}^{2-}\right.$ 、 $\mathrm{HCO}_{3}^{-}$) 和溶解性有机质 (DOM) 中不同浓度的胡敏酸 ( HA) 、富里酸 (FA) 和商品化腐殖酸 ( SHA), 研究其对 COD 和 $\mathrm{COD}_{\mathrm{Mn}}$ 测定的影响, 探讨 $\mathrm{Cl}^{-}$和 DOM 共同存在下对 $\mathrm{COD}$ 测定的影响. 结果表明: 程海水体中 $\mathrm{Cl}^{-}$浓度对 $\mathrm{COD}$ 存在显著影 响, 产生的 $\mathrm{COD}$ 值为 $5.42 \mathrm{mg} / \mathrm{L}, \mathrm{S}^{2-} 、 \mathrm{~F}^{-}$和 $\mathrm{HCO}_{3}^{-}$对 $\mathrm{COD}$ 影响较小; 各离子对 $\mathrm{COD}_{\mathrm{Mn}}$ 的影响很小; 不同浓度梯度的 $\mathrm{HA} 、 \mathrm{FA}$

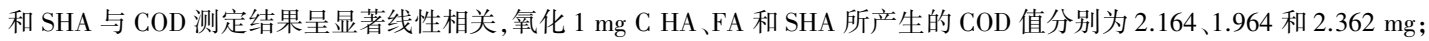
氧化 $1 \mathrm{mg}$ C HA 和 FA 所产生的 $\mathrm{COD}_{\mathrm{Mn}}$ 值分别为 0.646 和 $0.344 \mathrm{mg}$; DOM 对 $\mathrm{COD}$ 测定值的影响显著大于对 $\mathrm{COD}_{\mathrm{Mn}}$ 测定值 的影响; 且一定浓度 $\mathrm{Cl}^{-}$的存在增强了 $\mathrm{FA}$ 与 $\mathrm{HA}$ 对 $\mathrm{COD}$ 测定的影响. 该研究为进一步阐明程海 $\mathrm{COD}$ 逐步升高, $\mathrm{COD}_{\mathrm{Mn}}$ 值 缓慢升高的内在原因奠定了基础.
\end{abstract}

关键词: 化学需氧量; 氯离子; 胡敏酸;富里酸;程海

\section{Effects of inorganic anions and dissolved organic matter on COD measurement in Lake Chenghai}

BI Tingting, YANG Benqin *** LUO Nao, DU Ya'nan, YANG Jinfeng, REN Dong, GU Lipeng \& PAN Xuejun (Faculty of Environmental Science and Engineering, Kunming University of Science and Technology, Kunming 650500, P.R. China)

\begin{abstract}
In Lake Chenghai, annual increase in chemical oxygen demand (COD) has been observed since 2005, while biochemical oxygen demand ( BOD) has remained almost constant and permanganate index $\left(\mathrm{COD}_{\mathrm{Mn}}\right)$ has been increasing slowly. In order to reveal the reason for the phenomenon, typical inorganic anions such as $\mathrm{Cl}^{-}, \mathrm{F}^{-}, \mathrm{S}^{2-}$ and $\mathrm{HCO}_{3}^{-}$, as well as dissolved organic matter (DOM) such as humic acid (HA), fulvic acid (FA) and commercial humic acid (SHA) in Lake Chenghai were surveyed for the effect of COD measurement. This study investigated the effects of inorganic anions and major DOM fractions, and their combined occurrences on COD measurement. Results showed that: $\mathrm{Cl}^{-}$could significantly affect the COD measurement with the value of $5.42 \mathrm{mg} / \mathrm{L}$, while the presence of $\mathrm{S}^{2-}, \mathrm{F}^{-}$and $\mathrm{HCO}_{3}^{-}$may hardly impair accuracy of $\mathrm{COD}$ measurement; the inorganic anions also had a less impact on $\mathrm{COD}_{\mathrm{Mn}}$ measurement. The different concentrations of HA, FA, SHA were significantly linearly correlated with COD values, the oxidation of $1 \mathrm{mg} \mathrm{C} \mathrm{HA,} \mathrm{FA} \mathrm{and} \mathrm{SHA} \mathrm{produced} \mathrm{2.164,} 1.964$ and $2.362 \mathrm{mg}$ COD, respectively; while the oxidation of $1 \mathrm{mg}$ C HA and FA produced 0.646 and $0.344 \mathrm{mg} \mathrm{COD}_{\mathrm{Mn}}$, respectively. The influence of DOM on COD measurement was significantly larger than that of $\mathrm{COD}_{\mathrm{Mn}}$; and the different concentrations of chloride ions enhanced the effect of FA and HA on COD determination. This study can build up the foundation for further exploring the internal causes for the increase in COD and COD in Lake Chenghai.
\end{abstract}

Keywords: Chemical oxygen demand; chloride ions; humic acid; fulvic acid; Lake Chenghai

化学需氧量 (COD) 是指在一定条件下, 用强氧化剂处理水量时所消耗氧化剂的量, 是反映水体受还原

* 云南环境保护专项资助. 2016-10-20 收稿; 2017-02-21 收修改稿. 毕婷婷( 1991 ), 女, 硕士研究生; E-mail: ttingbi@163.com.

** 通信作者; E-mail: ynybq87@ kmust.edu.cn. 
性物质污染程度的重要指标, 也是衡量水体中有机物含量的重要指标. 溶解性有机质 (DOM) 是地表水体中 普遍存在的活跃组分 ${ }^{[1]}$, 主要来源于水体微生物及藻类降解和土壤及动植物有机质的残体 ${ }^{[2]}$, 其主要成分 为腐殖酸. 腐殖酸包括胡敏酸 $(\mathrm{HA})$ 和富里酸 $(\mathrm{FA})$, 以溶解性有机碳含量计, 腐殖酸的含量可占到环境地表 水中溶解性有机碳总量的 $40 \% \sim 80 \%{ }^{[3]}$. 天然水体中总有机碳的浓度范围为 $1 \sim 40 \mathrm{mg} / \mathrm{L}^{[4]}$, 江河中通常可达 到 $7 \mathrm{mg} / \mathrm{L}^{[5]}$.

天然水体中存储了大量无机离子和溶解性有机质,其对水体 COD 值会产生影响. 先前研究表明, 理论 上氧化 $1 \mathrm{mg} \mathrm{Cl}^{-}$所产生的 $\mathrm{COD}$ 值为 $0.226 \mathrm{mg}^{[6-7]}$, 氧化 $1 \mathrm{mg} \mathrm{S}^{2-}$ 为 $\mathrm{SO}_{4}^{2-}$ 需 $2 \mathrm{mg}$ 氧 ${ }^{[8]}$. 郭玉文等 ${ }^{[9]}$ 曾提到: 根据 $\mathrm{Fe}^{2+}$ 与 $\mathrm{K}_{2} \mathrm{Cr}_{2} \mathrm{O}_{7}$ 的反应, 可计算出氧化 $1 \mathrm{~g} \mathrm{Fe}^{2+}$ 的理论需氧量为 $0.11 \mathrm{~g}$; 谢文玉等 ${ }^{[10]}$ 研究表明在 $0 \sim 200 \mathrm{mg} / \mathrm{L}$ $\mathrm{NO}_{2}^{-}$范围内, $\mathrm{NO}_{2}^{-}$与 $\mathrm{COD}$ 值有良好的线性关系, $1 \mathrm{mg} \mathrm{NO}_{2}^{-}$相当于 $0.3458 \mathrm{mg} \mathrm{COD}$. 因此, 无机离子对水体 $\mathrm{COD}$ 存在一定的影响. 同时日本学者对 Biwa 湖水质进行长期监测, 发现湖水中 COD 自 1985 年起呈不断增加的 趋势, 而且溶解性有机质部分占 COD 的主要组成部分 ${ }^{[11-14]}$, 不能被微生物利用的部分难降解 DOM 的积累 导致了湖泊 COD 的增加 ${ }^{[15]}$. 韩国的 Hang 河和 Paldang 湖也出现了因为难降解有机质的积累而导致 COD 增 加的现象 ${ }^{[16-17]}$. 故 DOM 对 COD 存在显著的影响.

程海 $\left(26^{\circ} 27^{\prime} \sim 26^{\circ} 38^{\prime} \mathrm{N}, 100^{\circ} 38^{\prime} \sim 100^{\circ} 41^{\prime} \mathrm{E}\right.$ ) 作为云南省九大高原湖泊之一, 滇西第二大淡水湖, 水体交 换周期较长, 蒸发量大, 地表径流补给不丰, 年内干湿季节转换明显 ${ }^{[18-19]}$; 流域水资源和水环境承载力以及 自净能力相对较小, 浮游植物种类繁多, 营养物质积累, 水生生态系统比较脆弱 ${ }^{[20]}$. 程海水体中 $\mathrm{Cl}^{-} 、 \mathrm{~F}^{-}$和 $\mathrm{HCO}_{3}^{-}$浓度较高, 而其他的还原性离子如 $\mathrm{S}^{2-} 、 \mathrm{Fe}^{2+}$ 等浓度相对较低. 自 2005 年以来, 程海水体的 $\mathrm{COD}$ 持续升 高, 而生化需氧量 $(\mathrm{BOD})$ 却维持不变, 高锰酸盐指数 $\left(\mathrm{COD}_{\mathrm{Mn}}\right)$ 升高也较缓慢, 因此对其 $\mathrm{COD}$ 持续升高原因 的探究就成为一项重要工作.

因此, 在探究程海 $\mathrm{COD}$ 持续升高的过程中, 研究程海水体中 $\mathrm{COD}$ 的变化是否与水体中存储的大量有机 质及各种无机还原性离子有关, 了解 DOM 与水体中其他无机还原性离子共存时对 COD 的影响是否存在交 互作用而导致 COD 进一步升高至关重要. 基于此, 本研究以自然水体中的无机还原性离子以及 DOM 为研 究对象, 研究无机还原性离子、DOM 以及二者共同存在时对 COD 测定的影响, 以探讨二者在 COD 持续升高 过程中的贡献.

\section{1 材料与方法}

\section{1 仪器与试剂}

仪器: 德国 ElementarVario TOC cube (用于测定 DOM 的浓度), 四川优普 UPH II 型超纯水器 (电阻率> $18.2 \mathrm{M} \Omega \cdot \mathrm{cm}$ ), 湖南湘仪 L550 低速离心机, 日本 Tokyo Rikakikai 产 FDU-1200 冷冻干燥仪, 美国 Denver 产 TB-214 精密电子天平 (精度 $0.01 \mathrm{mg}$ ), $33 \mathrm{~cm}$ 球形冷凝管 $\mathrm{COD}$ 回流装置 (用于 COD 测定的消解过程).

试剂: $\left(\mathrm{NH}_{4}\right)_{2} \mathrm{SO}_{4} \cdot \mathrm{FeSO}_{4} \cdot 6 \mathrm{H}_{2} \mathrm{O} 、 \mathrm{C}_{12} \mathrm{H}_{8} \mathrm{~N}_{2} \cdot \mathrm{H}_{2} \mathrm{O} 、 \mathrm{Ag}_{2} \mathrm{SO}_{4} 、 \mathrm{NaOH} 、 \mathrm{KMnO}_{4}$ 和 $\mathrm{Na}_{2} \mathrm{C}_{2} \mathrm{O}_{4}$ 均为购于 Aladdin 的分析纯试剂, $\mathrm{NaCl} 、 \mathrm{NaF} 、 \mathrm{Na}_{2} \mathrm{~S}$ 和 $\mathrm{NaHCO}_{3}$ 均为购于国药集团化学试剂有限公司的分析纯试剂, 优级纯的浓 $\mathrm{H}_{2} \mathrm{SO}_{4}$ 购于国药集团化学试剂有限公司, 优级纯的 $\mathrm{K}_{2} \mathrm{Cr}_{2} \mathrm{O}_{7} 、 \mathrm{DAX}-8$ 大孔树脂和商品化腐殖酸 ( $\mathrm{SHA}$ ) 均购买 自 Sigma Aldrich.

\section{2 无机盐溶液的配置}

选取程海水体中具有代表性的无机阴离子 $\left(\mathrm{Cl}^{-} 、 \mathrm{~F}^{-} 、 \mathrm{~S}^{2-} 、 \mathrm{HCO}_{3}^{-}\right)$为研究对象. 云南省环境监测中心站监 测数据显示, 程海水体中 $\mathrm{Cl}^{-}$平均浓度为 $22.52 \mathrm{mg} / \mathrm{L}, \mathrm{F}^{-}$平均浓度为 $2.42 \mathrm{mg} / \mathrm{L}, \mathrm{S}^{2-}$ 平均浓度为 $0.17 \mathrm{mg} / \mathrm{L}$, $\mathrm{HCO}_{3}^{-}$平均浓度为 $470 \mathrm{mg} / \mathrm{L}$. 故分别称取 $\mathrm{NaCl} 、 \mathrm{NaF} 、 \mathrm{Na}_{2} \mathrm{~S}$ 和 $\mathrm{NaHCO}_{3}$ 药品溶于超纯水中, 保持相同的 $\mathrm{pH}$ 和 离子强度, 使各离子浓度分别为 $\mathrm{Cl}^{-}: 0 、 2 、 5 、 10 、 15 、 20 、 25 、 30 、 35 、 40 、 45 、 50 \mathrm{mg} / \mathrm{L} ; \mathrm{S}^{2-}: 0 、 0.1 、 1 、 5 、 10 、 20 、 30 、$ $40 \mathrm{mg} / \mathrm{L} ; \mathrm{F}^{-}: 0 、 1 、 2 、 3 、 5 、 10 、 15 、 30 、 100 \mathrm{mg} / \mathrm{L} ; \mathrm{HCO}_{3}^{-}: 0 、 100 、 200 、 300 、 400 、 500 、 600 \mathrm{mg} / \mathrm{L}$.

\section{3 腐殖酸的提取及配置}

将从 Sigma 上购买的 SHA 按照国际腐殖酸协会 (International Humic Substance Society, IHSS) 推荐的方 法, 将沉积物与超纯水按 $1: 10$ 的比例混合 ${ }^{[21]}$, 用 $\mathrm{H}_{2} \mathrm{SO}_{4}$ 和 $\mathrm{NaOH}$ 通过酸沉降和碱提取进一步纯化 SHA. 同 时采用实验室从湖泊中已分离纯化的 FA 和 HA 进行实验. 
分别称取 $0.50 \mathrm{~g}$ 提纯的 HA、FA 和 SHA 于具塞雉形瓶中, 分别向 HA 和 SHA 中加人 $500 \mathrm{ml} 0.05 \mathrm{~mol} / \mathrm{L}$ $\mathrm{NaOH}$ 溶液, $\mathrm{FA}$ 中加人 $500 \mathrm{ml}$ 超纯水, 于 150 次 $/ \mathrm{min}$ 频率下震动 $24 \mathrm{~h}$, 经预先灼烧 $4 \mathrm{~h}$ 的 $0.45 \mu \mathrm{m}$ 的玻璃纤 维滤膜抽滤后作为 HA、FA 和 SHA 的母液. 测定溶解性有机碳浓度后于 $4^{\circ} \mathrm{C}$ 下避光保存, 以免其发生降 解 ${ }^{[22]}$. 将各母液稀释至 $2 、 5 、 8 、 10 、 15 、 20 、 30 \mathrm{mg} \mathrm{C} / \mathrm{L}$ 的浓度梯度进行研究.

\section{$1.4 \mathrm{COD}$ 的测定方法}

采用 GB 11914-1989 中的重铬酸钾法对 COD 进行测定; 采用 GB 11892-1989 中的酸性法对 $\mathrm{COD}_{\mathrm{Mn}}$ 进 行测定. 通过 SPSS 20.0、Origin 9.0 等软件对实验结果进行分析.

\section{2 结果与讨论}

\section{1 无机阴离子对 COD 测定的影响}

2.1.1 不同无机阴离子单独存在时对 COD 测定的影响 含有各种无机阴离子的溶液稀释至不同浓度梯度, 采用低浓度 $\mathrm{COD}$ 的重铬酸钾法, 对其 COD 进行测定, 结果如图 1 所示.

研究结果表明: 当 $\mathrm{Cl}^{-}$浓度小于 $50 \mathrm{mg} / \mathrm{L}$ 时,一定浓度梯度下的 $\mathrm{Cl}^{-}$与 $\mathrm{COD}$ 测定结果值相关系数可达到 0.999 , 呈显著性相关 (图 1a). 回归分析曲线为: $\mathrm{COD}=-0.0034\left(\mathrm{Cl}^{-}\right)^{2}+0.3173 \mathrm{Cl}^{-}-0.0001, R^{2}=0.9978$. 当 $\mathrm{Cl}^{-}$ 浓度小于 $30 \mathrm{mg} / \mathrm{L}$ 时, 回归分析曲线为: $\mathrm{COD}=0.2194 \mathrm{Cl}^{-}+0.3454, R^{2}=0.9768 . \mathrm{S}^{2-}$ 与 $\mathrm{COD}$ 相关系数为 0.998 , 呈显著线性相关, 回归分析曲线为: $\mathrm{COD}=1.7427 \mathrm{~S}^{2-}-0.044, R^{2}=0.999$ (图 1b).一定浓度梯度下的 $\mathrm{F}^{-}$与 $\mathrm{COD}$ 测定结果值相关系数为 0.291 (图 1c). $\mathrm{HCO}_{3}^{-}$与 $\mathrm{COD}$ 测定结果值相关系数为 0.517 , 相关性较弱 (图 1d).
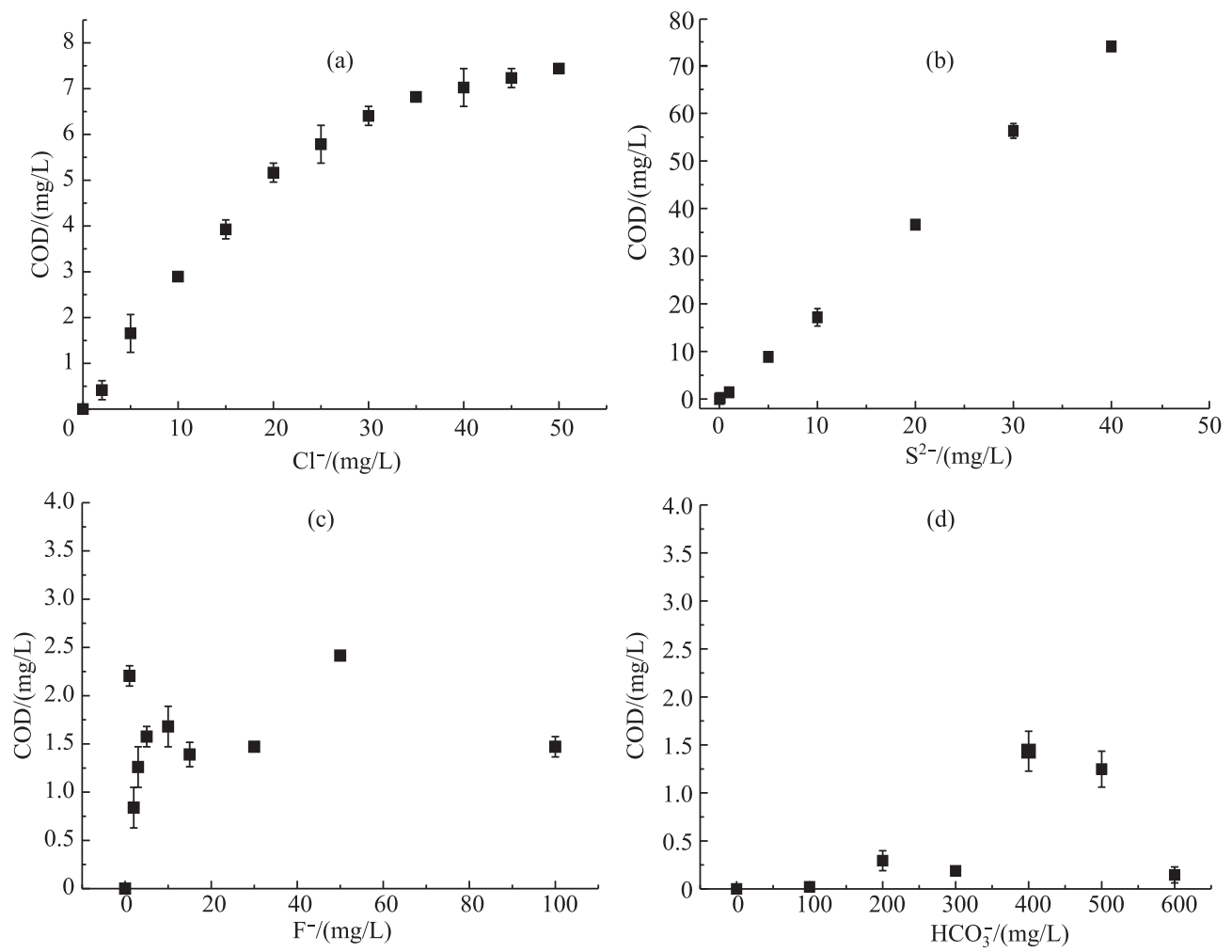

图 1 不同浓度梯度的 $\mathrm{Cl}^{-}(\mathrm{a}) 、 \mathrm{~S}^{2-}(\mathrm{b}) 、 \mathrm{~F}^{-}(\mathrm{c}) 、 \mathrm{HCO}_{3}^{-}(\mathrm{d})$ 与 $\mathrm{COD}$ 的关系

Fig. 1 The relationship between different concentrations of $\mathrm{Cl}^{-}(\mathrm{a}), \mathrm{S}^{2-}(\mathrm{b}), \mathrm{F}^{-}(\mathrm{c}), \mathrm{HCO}_{3}^{-}(\mathrm{d})$ and $\mathrm{COD}$

在 COD 测定的实验过程中, 根据重铬酸钾氧化反应及 $\mathrm{Cl}^{-}$反应的电极电位可知, 重铬酸钾完全可以氧 化 $\mathrm{Cl}^{-}$. 图 1a 表明, 当 $\mathrm{Cl}^{-}$浓度小于 $30 \mathrm{mg} / \mathrm{L}$ 时, 实验结果与理论值较为接近. 当 $\mathrm{Cl}^{-}$浓度大于 $30 \mathrm{mg} / \mathrm{L}$ 时, 
$\mathrm{COD}$ 值的增长趋势变缓, 且实验过程中有白色沉淀生成. 原因是 $\mathrm{Cl}^{-}$可以消耗催化剂 $\mathrm{Ag}_{2} \mathrm{SO}_{4}$, 水样中 $\mathrm{Cl}^{-}$浓 度较高, 部分未被重铬酸钾氧化的 $\mathrm{Cl}^{-}$与 $\mathrm{Ag}_{2} \mathrm{SO}_{4}$ 反应生成 $\mathrm{AgCl}$ 沉淀, 从而消耗 $\mathrm{Ag}_{2} \mathrm{SO}_{4}$, 降低反应速率, $\mathrm{COD}$ 值增长趋势变缓. 程海水体中, $\mathrm{Cl}^{-}$平均浓度为 $22.52 \mathrm{mg} / \mathrm{L}$, 浓度较高, 其对程海 $\mathrm{COD}$ 测定存在显著影响, 产 生的 $\mathrm{COD}$ 值为 $5.42 \mathrm{mg} / \mathrm{L} . \mathrm{S}^{2-}$ 亦能被重铬酸钾氧化成 $\mathrm{SO}_{4}^{2-}$, 理论上氧化 $1 \mathrm{mg} \mathrm{\textrm {S } ^ { 2 - }}$ 生成 $\mathrm{SO}_{4}^{2-}$ 需 $2 \mathrm{mg}$ 氧 ${ }^{[8]}$, 实 验结果与理论值相比略小, 特别是 $\mathrm{S}^{2-}$ 浓度低于 $5 \mathrm{mg} / \mathrm{L}$ 时, 影响很小, 这与 $\mathrm{S}^{2-}$ 极容易被空气氧化有关, 使实 验结果与理论值存在偏差. 程海水体中, $\mathrm{S}^{2-}$ 浓度较低, 平均浓度为 $0.17 \mathrm{mg} / \mathrm{L}$, 此浓度下其对 $\mathrm{COD}$ 贡献很小, 故认为 $\mathrm{S}^{2-}$ 对程海 $\mathrm{COD}$ 的影响较小. 由于实验方法为加热消解, 在酸性条件下 $\mathrm{HCO}_{3}^{-}$易生成 $\mathrm{CO}_{2}$ 气体, 故对 $\mathrm{COD}$ 的影响较小. $\mathrm{F}^{-}$与 $\mathrm{COD}$ 的实验结果值相关性较弱, 认为其对水体中 COD 的影响较小, 可以忽略不计.

2.1.2 混合无机阴离子对 COD 测定的影响 模拟程海水体中各还原性无机阴离子的浓度, 配制混合离子溶 液 $\left(\mathrm{Cl}^{-}: 20 \mathrm{mg} / \mathrm{L} ; \mathrm{F}^{-}: 2.50 \mathrm{mg} / \mathrm{L} ; \mathrm{HCO}_{3}^{-}: 450 \mathrm{mg} / \mathrm{L} ; \mathrm{S}^{2-}: 0.20 \mathrm{mg} / \mathrm{L} ; \mathrm{Fe}^{2+}: 0.02 \mathrm{mg} / \mathrm{L} ; \mathrm{NO}_{2}^{-}: 0.005 \mathrm{mg} / \mathrm{L}\right.$ ), 其 COD 测定值为 $5.36 \mathrm{mg} / \mathrm{L}$, 与 $\mathrm{Cl}^{-}$浓度对 $\mathrm{COD}$ 测定值的结果一致. 相对于各混合离子对 $\mathrm{COD}$ 测定影响而言, $\mathrm{Cl}^{-}$仍 然是对 COD 测定值影响的显著因素.

将邻苯二甲酸氢钾配制成 COD 标准溶液, 加人等浓度的上述无机阴离子, 其对 COD 测定值的影响与 $\mathrm{Cl}^{-}$对 $\mathrm{COD}$ 测定值的影响相似.

2.1.3 $\mathrm{Cl}^{-}$对 $\mathrm{COD}_{\mathrm{Mn}}$ 测定的影响 将含有 $\mathrm{Cl}^{-}$的溶液稀释至不同浓度梯度 $(10 、 20 、 30 、 40 、 50 、 60 \mathrm{mg} / \mathrm{L})$, 采用酸 性高镇酸盐指数法, 对 $\mathrm{COD}_{\mathrm{Mn}}$ 进行测定, $\mathrm{COD}_{\mathrm{Mn}}$ 值分别为 $0.49 、 0.49 、 0.59 、 0.47 、 0.53$ 和 0.57 , 表明 $\mathrm{Cl}^{-}$对 $\mathrm{COD}_{\mathrm{Mn}}$ 的影响很小, 可以忽略不计. 故程海水体中还原性无机阴离子的存在增加了水质的 $\mathrm{COD}$ 值, 但对 $\mathrm{COD}_{\mathrm{Mn}}$ 的影响很小, 可能是导致程海水体 $\mathrm{COD}$ 持续升高, $\mathrm{COD}_{\mathrm{Mn}}$ 缓慢升高的原因之一.

\section{2 溶解性有机质对 $C O D 、 C O D_{\mathrm{Mn}}$ 测定的影响}

不同浓度梯度的 HA、FA 和 SHA 与 COD 测定结果值相关系数均大于 0.99 , 均呈显著线性相关 (图 2a), 回归分析曲线分别为: $\mathrm{COD}=2.1816 \mathrm{HA}-0.3403, R^{2}=0.9994 ; \mathrm{COD}=1.9883 \mathrm{FA}-0.4666, R^{2}=0.9995 ; \mathrm{COD}=$ $2.2952 \mathrm{SHA}+1.2726, R^{2}=0.9965$. 经坐标原点修正后, 即氧化 $1 \mathrm{mg}$ C HA 所产生的 COD 值为 $2.164 \mathrm{mg}$, 氧化 1

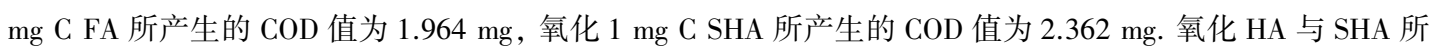
产生的 COD 值相近, 氧化 HA 所产生的 COD 值高于氧化 FA 所产生的 COD 值. 不同浓度梯度的 HA 和 FA 与 $\mathrm{COD}_{\mathrm{Mn}}$ 测定结果值也均呈显著线性相关 (图 $2 \mathrm{~b}$ ), 回归分析曲线分别为: $\mathrm{COD}_{\mathrm{Mn}}=0.6457 \mathrm{HA}+0.0097, R^{2}=$ $0.9991 ; \mathrm{COD}_{\mathrm{Mn}}=0.3312 \mathrm{FA}+0.2366, R^{2}=0.9955$. 经坐标原点修正后, 即氧化 $1 \mathrm{mg} \mathrm{C} \mathrm{HA}$ 所产生的 $\mathrm{COD}_{\mathrm{Mn}}$ 值为 $0.646 \mathrm{mg}$, 氧化 $1 \mathrm{mg}$ C FA 所产生的 COD 值为 $0.344 \mathrm{mg}$. 氧化 HA 所产生的 $\mathrm{COD}_{\mathrm{Mn}}$ 值高于氧化 FA 所产生的 $\mathrm{COD}_{\mathrm{Mn}}$ 值, 与 $\mathrm{HA}$ 和 $\mathrm{FA}$ 与 $\mathrm{COD}$ 测定的影响一致. 同时 $\mathrm{HA}$ 和 $\mathrm{FA}$ 对 $\mathrm{COD}$ 测定值的影响大于对 $\mathrm{COD}_{\mathrm{Mn}}$ 的影 响, 这与重铬酸钾的氧化性强于高锰酸钾的氧化性有关. 故研究表明 DOM 可能是导致程海水体 COD 持续 升高, $\mathrm{COD}_{\mathrm{Mn}}$ 缓慢升高的原因之一.
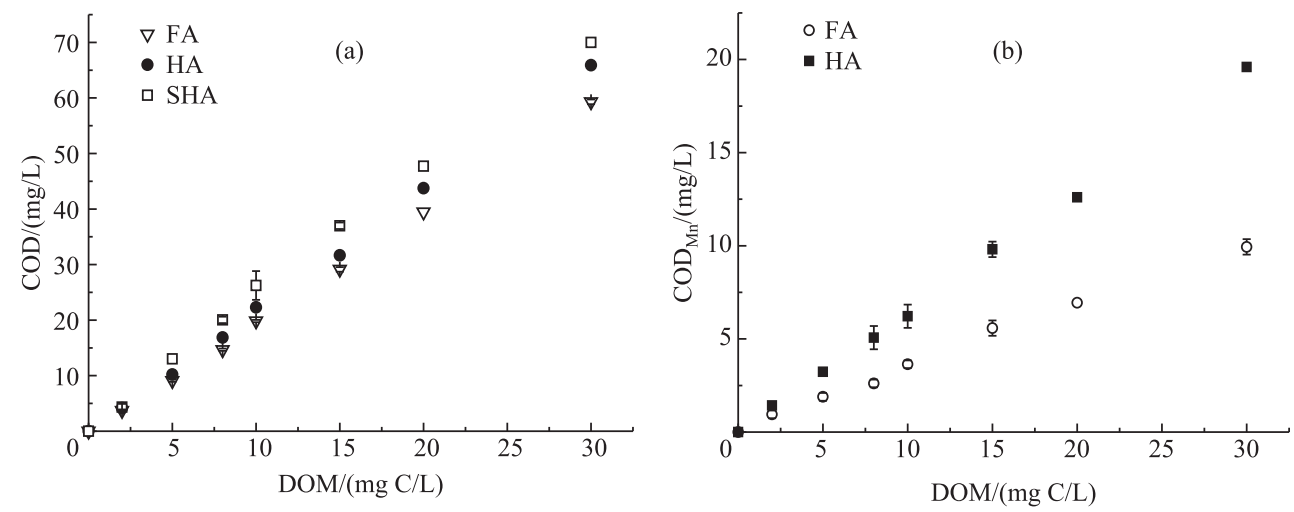

图 2 不同浓度的 $\mathrm{DOM}$ 与 $\operatorname{COD}(\mathrm{a})$ 和 $\mathrm{COD}_{\mathrm{Mn}}(\mathrm{b})$ 的关系

Fig.2 The relationship between $\operatorname{COD}(\mathrm{a}), \mathrm{COD}_{\mathrm{Mn}}(\mathrm{b})$ and different concentrations of DOM 


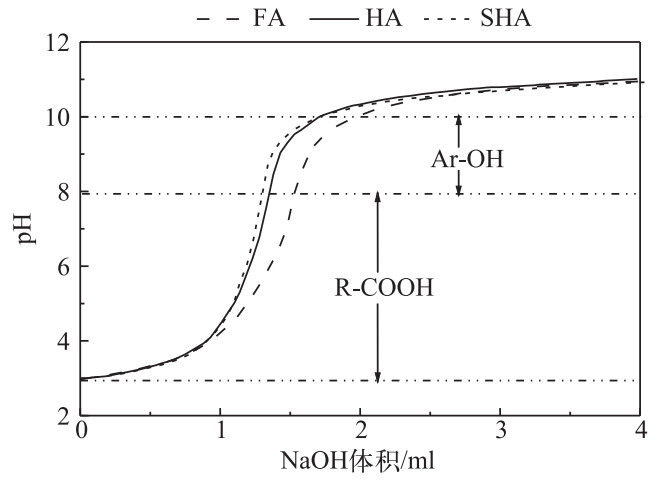

图 3 官能团滴定结果

Fig. 3 The results of functional group titration
Myneni 等 ${ }^{[23]}$ 的 13C-NMR 谱图揭示 HA 结构中含 有大量的长链烷烃,一定量的芳香烃、羧基和碳水化合 物, $\mathrm{FA}$ 则含有大量的羧基、碳水化合物及多糖, 芳香基 和烷基为其主要结构特征. 水环境中腐殖质具有苯环 羧基和酚羟基官能团构成的聚苯环,所含官能团主要有 羧基、醇羟基和酚羟基等,其中羧基约占总酸性基团的 $60 \% \sim 90 \%{ }^{[24]}$. 官能团滴定结果 (图 3) 显示, FA 的羧基 及羟基的含量高于 HA 和 SHA 中羧基及羟基的含量, 这与 $\mathrm{Ma}$ 等 ${ }^{[25]}$ 对官能团滴定的实验结果一致. 含羟基的 有机化合物在强酸性介质中首先被重铬酸钾氧化为羧 酸,生成的脂肪酸与硫酸银生成脂肪酸根, 由于银离子 的作用,使羧基易断裂而生成 $\mathrm{CO}_{2}$ 和 $\mathrm{H}_{2} \mathrm{O}$, 并进一步生 成新的脂肪酸根, 其较前者少 1 个碳原子. 如此循环重 复,逐步使有机物全部氧化成 $\mathrm{CO}_{2}$ 和 $\mathrm{H}_{2} \mathrm{O}$. 若 DOM 中羟

基含量越多, 越容易被氧化. FA 相较于 HA 和 SHA 含有更多的羟基官能团, 则氧化 HA 和 SHA 比氧化 FA 所需要消耗的氧含量多, 即氧化 HA 和 SHA 比氧化 FA 所产生的 COD 值高, 与之前的实验结果一致.

\section{$2.3 \mathrm{Cl}^{-}$与 DOM 同时存在时对 COD 测定的影响}

程海水体中, $\mathrm{Cl}^{-}$浓度平均值为 $22.52 \mathrm{mg} / \mathrm{L}$, 且相对较稳定, 选用 $\mathrm{Cl}^{-}$与 $\mathrm{DOM}$ 质相混合, 研究其对 COD 测定 的影响. 考虑到天然水体中总有机碳浓度范围为 $1 \sim 40 \mathrm{mg} / \mathrm{L}$, 故设定 $\mathrm{Cl}^{-}$浓度分别为 $2 、 5 、 10 、 20$ 和 $30 \mathrm{mg} / \mathrm{L}, \mathrm{FA}$ 和 HA 浓度分别为 $2 、 5 、 10 、 15 、 20$ 和 $30 \mathrm{mg} \mathrm{C} / \mathrm{L}$. 其实验结果如图 4 所示.
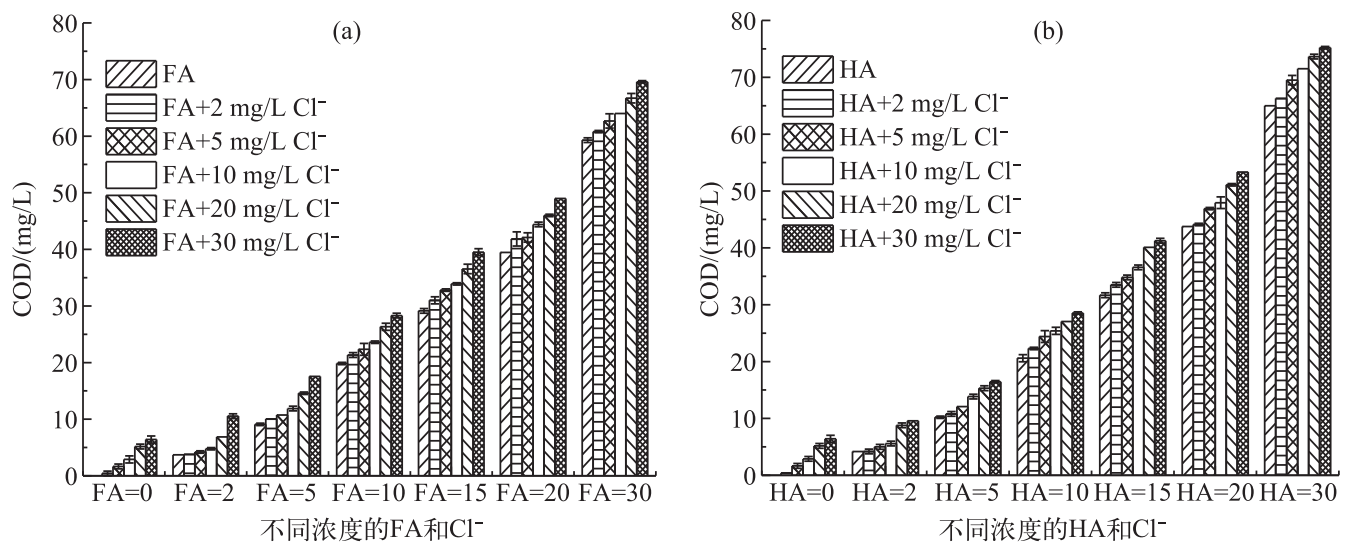

图 4 不同浓度的 $\mathrm{FA}(\mathrm{a}) 、 \mathrm{HA}(\mathrm{b})$ 和 $\mathrm{Cl}^{-}$与 $\mathrm{COD}$ 的关系

Fig.4 The relationship between COD and different concentrations of $\mathrm{FA}+\mathrm{Cl}^{-}(\mathrm{a})$ and $\mathrm{HA}+\mathrm{Cl}^{-}(\mathrm{b})$

$\mathrm{FA} 、 \mathrm{HA}$ 浓度一定时, 随着 $\mathrm{Cl}^{-}$浓度的升高, $\mathrm{COD}$ 值随之升高, 并呈良好的线性关系, 且当 $\mathrm{Cl}^{-}$浓度一定 时, 随着 FA、 $\mathrm{HA}$ 浓度的升高, COD 值也随之升高加. 混合溶液的 COD 值相对于 $\mathrm{FA} 、 \mathrm{HA}^{-}$和 $\mathrm{Cl}^{-}$单独存在时的 COD 值较大. FA、HA 的浓度越大, COD 值升高程度也越大, 其对 COD 的影响也呈上升趋势 (图 4). SPSS 分 析结果显示, $\mathrm{Cl}^{-}$与 $\mathrm{FA} 、 \mathrm{HA}$ 分别呈显著的交互作用, 表明 $\mathrm{Cl}^{-}$的存在使 $\mathrm{FA}$ 与 $\mathrm{HA}$ 对 $\mathrm{COD}$ 的影响产生差异. 随 着 $\mathrm{Cl}^{-}$浓度的增大, $\mathrm{FA}$ 和 $\mathrm{HA}$ 与 $\mathrm{COD}$ 的线性关系的斜率逐渐增大, 表明随着 $\mathrm{Cl}^{-}$浓度升高, 氧化 $\mathrm{FA}$ 和 $\mathrm{HA}$ 所 产生的 COD 值逐渐升高, 其对 COD 的影响呈逐渐增大的趋势 (图 4). 即一定浓度的 $\mathrm{Cl}^{-}$的存在增强了 $\mathrm{FA}$ 与 HA 对 COD 的影响. 由于 HA 和 FA 都属于结构复杂的混合物, 尚不明确其具体结构, 其对 COD 的影响有待 于进一步研究. 


\section{3 结论}

1) 程海水体中具有代表性的无机阴离子 $\left(\mathrm{Cl}^{-} 、 \mathrm{~F}^{-} 、 \mathrm{~S}^{2-} 、 \mathrm{HCO}_{3}^{-}\right)$中, $\mathrm{Cl}^{-}$对 $\mathrm{COD}$ 测定存在显著影响, $\mathrm{S}^{2-} 、 \mathrm{~F}^{-}$ 和 $\mathrm{HCO}_{3}^{-}$对 $\mathrm{COD}$ 测定影响较小, 对 $\mathrm{COD}_{\mathrm{Mn}}$ 的影响也较小.

2) 不同浓度的 $\mathrm{HA} 、 \mathrm{FA}$ 和 $\mathrm{SHA}$ 与 $\mathrm{COD}$ 和 $\mathrm{COD}_{\mathrm{Mn}}$ 测定结果均呈显著线性相关. 即氧化 $1 \mathrm{mg} \mathrm{C} \mathrm{HA} 、 \mathrm{FA}$ 和

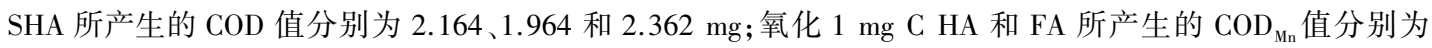
0.646 和 $0.344 \mathrm{mg}$. FA 相较于 HA 含有更多的差基官能团, 氧化 HA 比氧化 FA 所产生的 COD 值高. DOM 对 $\mathrm{COD}$ 和 $\mathrm{COD}_{\mathrm{Mn}}$ 测定值存在显著影响, 且对 $\mathrm{COD}$ 值的影响显著大于对 $\mathrm{COD}_{\mathrm{Mn}}$ 值的影响.

3) 随着 $\mathrm{Cl}^{-}$浓度的增大, 氧化 $\mathrm{FA}$ 和 HA 所产生的 $\mathrm{COD}$ 值逐渐增高, 对 $\mathrm{COD}$ 测定的影响呈逐渐增大的趋 势. 一定浓度 $\mathrm{Cl}^{-}$的存在增强了 $\mathrm{FA}$ 与 $\mathrm{HA}$ 对 $\mathrm{COD}$ 测定的影响.

4) $\mathrm{Cl}^{-}$和 DOM 的存在是导致程海近年来 COD 逐渐升高的原因之一, 该研究为进一步阐明程海 COD 逐 步升高, $\mathrm{COD}_{\mathrm{Mn}}$ 值缓慢升高的内在原因奠定了基础,其机理机制有待进一步研究.

\section{4 参考文献}

[ 1 ] Kang S, Xing B. Humic acid fractionation upon sequential adsorption onto goethite. Langmuir, 2008, 24(6) : 2525-2531. DOI : $10.1021 /$ la702914q.

[ 2 ] Hur J, Park MH, Schlautman MA. Microbial transformation of dissolved leaf litter organic matter and its effects on selected organic matter operational descriptors. Environmental Science and Technology, 2009, 43 (7) : 2315-2321. DOI: 10. 1021/es802773b.

[ 3 ] Kitis M, Kilduff JE, Karanfil T. Isolation of dissolved organic matter (DOM) from surface waters using reverse osmosis and its impact on the reactivity of DOM to formation and speciation of disinfection by-products. Water Research, 2001, 35(9) : 2225-2234. DOI: 10.1016/S0043-1354(00) 00509-1.

[ 4 ] Thurman EM. Organic geochemistry of natural waters. Dordrecht: Martinus Nijho, 1985.

[ 5 ] Wu Fengchang, Wang Liying, Li Wen et al. Natural organic matter and its significance in terrestrial surface environment. $J$ Lake Sci, 2008, 20 (1) : 1-12. DOI:10.18307/2008.0101. [ 吴丰昌, 王立英, 黎文等. 天然有机质及其在地表环境中 的重要性. 湖泊科学, $2008,20(1): 1-12$.]

[ 6 ] Zhao Yaqian. Effects of chloride ammonia and hydrogen peroxide on COD measurement. Shanghai Environmental Sciences, 1995, 14 (8) : 32-34. [ 赵亚乾. $\mathrm{Cl}^{-}, \mathrm{NH}_{3}$ 和 $\mathrm{H}_{2} \mathrm{O}_{2}$ 对 $\mathrm{COD}$ 测定的影响. 上海环境科学, 1995, 14(8): 32-34.]

[ 7 ] Chen Lei, Jia Jinping. The different opinions of chloride ion's interference in the process of national standard method to determine the $\mathrm{COD}_{\mathrm{Cr}}$. Science \& Technology Information, 2013, (1) : 189-190. [陈砧, 贾金平. 重铬酸盐指数法测定 $\mathrm{COD}_{\mathrm{Cr}}$ 过程中氯离子干扰的疑问和探讨. 科技信息, 2013, (1) : 189-190.]

[ 8 ] Sun Dongyue, Guan Xiangyuan. Elimination of interference on COD measurement by $\mathrm{K}_{2} \mathrm{Cr}_{2} \mathrm{O}_{7}$ method. Environment Science and Management, 2009, 34(6) : 124-126. [孙冬月, 官香元. 重铬酸钾法测定 COD 中的干扰及消除. 环境科学 与管理, 2009, 34(6): 124-126.]

[ 9 ] Guo Yuwen, Yuan Dong. Discussion disturbing factors and its elimination in the determination of $\mathrm{COD}_{\mathrm{Cr}}$. Environmental Engineering, 2007, 25(6) : 73-74. [ 郭玉文, 袁东. 对 $\mathrm{COD}_{\mathrm{Cr}}$ 测定中干扰因素及其消除方法的探讨. 环境工程, $2007,25(6): 73-74$.

[10] Xie Wenyu, Chen Shaohua. Research on the elimination of nitrite interference in the determination of $\mathrm{COD}_{\mathrm{Cr}}$. Environmental Engineering, 2002, 20(5): 62-64. [谢文玉, 陈少华. 在 $\mathrm{COD}_{\mathrm{Cr}}$ 测定中消除亚硝酸根干扰的研究. 环境工程, $2002,20(5): 62-64$.

[11] Imai A, Fukushima T, Matsushige $\mathrm{K}$ et al. Fractionation of dissolved organic carbon from the waters of Lake Biwa and its inflowing rivers. Limnology, 1998, 59: 53-68. DOI:10.3739/rikusui.59.53.

[12] Imai A, Fukushima T, Matsushige $\mathrm{K}$ et al. Fractionation and characterization of dissolved organic matter in a shallow sutrophic lake, its inflowing rivers, and other organic matter sources. Pergamon, 2001, 17(35) : 4019-4028. DOI: 10.1016/ S0043-1354 ( 01 ) 00139-7.

[13 ] Hayakawa K. Seasonal variations and dynamics of dissolved carbohydrates in Lake Biwa. Organic Geochemistry, 2004,35 : 
169-179. DOI : 10.1016/j.orggeochem.2003.09.002.

[14] Hayakawa K. Research report of Lake Biwa. Environmental Research Institute, 2005, 22 : 161.

[15] Wada K, Yamanaka S, Yamamoto M et al. The characteristics and measuring technique of refractory dissolved organic substances in urban runoff. Water Research, 2006, 53(2) : 193-201. DOI:10.2166/wst.2006.053.

[16] Lee H, Choi J. Temporal analysis of trends in dissolved organic matter in Han River water. Environmental Engineering Research, 2009, 14(4) : 256-260. DOI: 10.4491/eer.2009.14.4.256.

[17] Nguyen HVM, Hur J. Tracing the sources of refractory dissolved organic matter in a large artificial lake using multiple analytical tools. Chemosphere, 2011, 85: 782-789. DOI:10.1016/j.chemosphere.2011.06.068.

[18] Cao Jinling, Xi Beidou, Xu Qigong et al. Regional differences in physiographical, climatological and morphological effects on lake trophic status in China. Acta Scientiae Circumstantiae, 2012, 32(6) : 199-206. [ 曹金玲, 席北斗, 许其功等. 地 理气候及湖盆形态对我国湖泊营养状态的影响. 环境科学学报, 2012, 32(6) : 199-206.]

[19] Dong Yunxian, Zou Rui eds. Study on ecosystem of Lake Chenghai. Kunming: Yunnan Science and Technology Press, 2010. [ 董云仙, 邹锐. 程海湖生态系统研究. 昆明: 云南科技出版社, 2010.]

[20] Dong Yunxian, Zhao Run, Tan Zhiwei. Eutrophication effect and ecological risk like Lake Chenghai. Annual conference of China Environmental Science Society (Chapter 4), 2014. [董云仙, 赵润, 谭志卫. 封闭型湖泊富营养化效应与生态 风险——程海为例. 2014 中国环境科学学会学术年会(第四章), 2014.]

[21] Bror OT. Dissolved organic matter characterization using multiday spectral decomposition of fluorescence landscape. Soil Science of America Journal, 2006, 70(6) : 2028-20377. DOI:10.2136/sssaj2006.0005.

[22] Ren Dong, Yang Xiaoxia, Ma Xiaodong et al. Structural characteristics of DOM and its effects on the photodeg-radation of 17ß-estradiol. China Environmental Science, 2015, 35(5): 1375-1383. [任东, 杨小霞, 马晓冬等. DOM 结构特征及 其对 17 $\beta$-雌二醇光降解的影响. 中国环境科学, 2015, 35(5): 1375-1383.]

[23] Myneni SCB, Brown JT, Martinez GA et al. Imaging of humic substance macromolecular structures in water and soils. Science, 1999, 286(5443) : 1335-1337. DOI:10.1126/science.286.5443.1335.

[24] Wu FC, Liu CQ. Humic substances. In: Leo ML, Nollet eds. Chromatographic analysis of the environment ( Third edition). Sound Parkway: CRC Press, 2006: 1155-1173.

[25] Ma H, Allen HE, Yin Y. Characterization of isolated fractions of dissolved organic matter from natural waters and a wastewater effluent. Water Research, 2001, 35(4) : 985-996. DOI:10.1016/S0043-1354( 00 )00350-X. 